\title{
Influence of weak anchoring on flow instabilities in nematic liquid crystals
}

\author{
O. S. Tarasov ${ }^{1,2}$, A. P Krekhov ${ }^{1,2}$ and L. Kramer ${ }^{1}$ \\ ${ }^{1}$ Institute of Physics, University of Bayreuth, D-95440 Bayreuth, Germany \\ ${ }^{2}$ Institute of Molecule and Crystal Physics, Russian Academy of Sciences, 450025 Ufa, Russia
}

(October 26, 2018)

\begin{abstract}
We analyse the homogeneous instabilities in a nematic liquid crystal subjected to plane steady Couette or Poiseuille flow in the case when the director is prealigned perpendicular to the flow plane taking into account weak anchoring at the confining surfaces. The critical shear rate decreases for decreasing anchoring strength and goes to zero in the limit of torque-free boundary conditions. For Poiseuille flow one has two types of instability depending on the values of the azimuthal $\left(W_{a}\right)$ and polar $\left(W_{p}\right)$ surface anchoring strengths. The critical line in $\left(W_{a}, W_{p}\right)$ space which separates the two instabilities regimes is obtained.
\end{abstract}

\section{INTRODUCTION}

During the last decade the study of surface anchoring in nematic liquid crystals (NLCs) for different types of confining substrates has attracted much attention. In particular, a change of anchoring strength strongly influences the orientational behaviour and dynamic response of NLCs under external electric and magnetic fields. This changes the switching times, which play an important role for applications (see, e.g., [1]). The hydrodynamic flow is a crucial ingredient for the dynamic response and switching characteristics of liquid crystal 
devices.

The anchoring characteristics can also be studied in orientational phenomena induced by hydrodynamic flow. In fact, recently the surface orientational transition caused by oscillatory shear flow was found [2] and the influence of weak anchoring on the linear response of the NLC to oscillatory flows was studied [3]. Up to now the study of orientational bulk instabilities in NLCs under hydrodynamic flow have been restricted to the case of strong surface anchoring (fixed director orientation at the confining plates) [4].

In this paper the influence of surface anchoring on the homogeneous instabilities in NLCs subjected to steady flow of Couette and Poiseuille type is studied theoretically in the case when the director at the bounding plates is oriented perpendicular to the flow plane. This is the simplest geometry because the initial state with the director oriented everywhere perpendicular to the flow plane is, by symmetry, a solution of the nematodynamic equations for any shear rate. The state can change only via a symmetry-breaking instability. The type of instability strongly depends on the sign of the Leslie viscosity coefficient $\alpha_{3}$. The case of negative $\alpha_{3}$ (flow-aligning materials) and strong anchoring of the director was investigated theoretically by Leslie [7] and Dubois-Violette and Manneville [5,8 10]. They have shown that in the absence of external fields the first instability is a homogeneous one in both, steady Couette [7,8] and Poiseuille [9] flows . The theoretical results were found to be in good agreement with experiments of Pieranski and Guyon and coworkers [1] 13. In Couette flow sufficiently strong additional magnetic field applied parallel to the initial director orientation was found to change the type of instability into a spatially periodic one where rolls develop 11, 13, in contrast to the case of Poiseuille flow, where a magnetic field does not change the type of instability [14]. Well above the threshold of the homogeneous instability in Poiseuille flow rolls were observed to develop in a secondary instability [15]. In the case of positive $\alpha_{3}$ (non-flow-aligning materials), according to the mechanism of Pieranski and Guyon, one has no homogeneous instability and only rolls are expected [11]. Since for these materials there is also a tumbling motion [16], the orientational behaviour of NLCs can be quite complex.

Here we focus on flow-aligning nematics. Starting from the standard set of nematody- 
namic equations in the Leslie-Ericksen formulation [4] the basic equations describing the homogeneous instabilities are presented (sec. II) taking into account arbitrary surface anchoring strength. Full semi-analytical solutions together with approximate expressions for the critical shear rate at which the homogeneous instabilities develop are obtained for steady Couette (sec. III) and Poiseuille (sec. IV) flow. In sec. $\nabla$ we discuss the results.

\section{BASIC EQUATIONS}

We consider a NLC layer of thickness $d$ sandwiched between two parallel infinite plates. The origin of a Cartesian coordinate system is placed at the centre of the layer with the $z$ axis perpendicular to the bounding plates. Steady Couette flow is generated by one plate (at $z=d / 2$ ) moving with constant velocity $V^{0}$ along the $x$ direction and the other plate (at $z=-d / 2$ ) fixed. Steady Poiseuille flow is obtained when a constant pressure gradient $\Delta P / \Delta x$ is applied along the $x$ axis. The confining plates provide a director orientation along the $y$ axis. The basic state is given by the stationary homogeneous solution of the standard set of nematodynamic equations (Leslie-Ericksen equations [4])

$$
\mathbf{n}^{0}=(0,1,0), \quad \mathbf{v}^{0}=\left(v_{x}^{0}, 0,0\right)
$$

where

$$
\begin{array}{ll}
v_{x}^{0}=V^{0}(1 / 2+z / d) & - \text { for Couette flow }, \\
v_{x}^{0}=-\frac{\Delta P}{\Delta x} \frac{d^{2}}{2 \eta_{3}}\left(1 / 4-z^{2} / d^{2}\right) & - \text { for Poiseuille flow },
\end{array}
$$

and $\eta_{3}=\alpha_{4} / 2$ with $\alpha_{4}$ the corresponding Leslie viscosity.

In order to investigate the stability of solution (1) we linearise the nematodynamic equations [4] with respect to perturbations that are homogeneous in the plane of the layer

$$
\mathbf{n}=\mathbf{n}^{0}+\left(n_{x}, n_{y}, n_{z}\right), \quad \mathbf{v}=\mathbf{v}^{0}+\left(v_{x}, v_{y}, v_{z}\right)
$$

where $n_{i}, v_{i}(i=x, y, z)$ are functions of $z$. We are looking for the existence of a stationary solution of the linearised nematodynamic equations, which signals the onset of a stationary (i.e. nonoscillatory) instability. The linearised equations are 


$$
\begin{array}{ll}
\eta_{1} v_{y, z z}+\left(\eta_{1}-\eta_{3}\right)\left(S n_{x}\right)_{, z} & =0, \\
\alpha_{2} S n_{z}-K_{22} n_{x, z z} & =0, \\
\alpha_{3} S n_{x}-K_{11} n_{z, z z}+\alpha_{3} v_{y, z} & =0,
\end{array}
$$

where $S=v_{x, z}^{0}$ is the shear rate, $\eta_{1}=\left(\alpha_{3}+\alpha_{4}+\alpha_{6}\right) / 2$ and $K_{11}, K_{22}$ are the elasticity coefficients for "splay" and "twist" deformations, respectively. The notation $f_{, z} \equiv d f / d z$ is used throughout.

The boundary conditions for the $y$-component of the velocity perturbation are

$$
v_{y}(z= \pm d / 2)=0
$$

The surface anchoring of the director is described in terms of a surface energy per unit area $F_{s}$ which has a minimum when the director at the surface is oriented along the easy axis (parallel to the $y$ axis in our case). It is convenient to characterise the surface anchoring by a "polar" anchoring strength $W_{p}$ pertaining to out-of-substrate-plane director deviations and an "azimuthal" anchoring strength $W_{a}$ related to distortions within the substrate plane. A phenomenological expression for the surface energy $F_{s}$ can be written in terms of an expansion with respect to $\left(\mathbf{n}-\mathbf{n}^{0}\right)$. For small director deviations from the easy axis one obtains for the surface energy

$$
F_{s}=\frac{1}{2} W_{a} n_{x}^{2}+\frac{1}{2} W_{p} n_{z}^{2}, \quad W_{a}>0, W_{p}>0
$$

The boundary conditions for the director perturbations can be obtained from the surface torques balance equation

$$
\pm K_{22} n_{x, z}+\frac{\partial F_{s}}{\partial n_{x}}=0, \quad \pm K_{11} n_{z, z}+\frac{\partial F_{s}}{\partial n_{z}}=0, \quad \text { for } z= \pm d / 2
$$

Introducing the dimensionless quantities

$$
\begin{aligned}
& \tilde{z}=\frac{z}{d}, \quad \tilde{S}=\beta \tau_{d} S, \quad \tau_{d}=\frac{\left(-\alpha_{2}\right) d^{2}}{K_{22}} \\
& V_{y}=\frac{\beta^{2} b \tau_{d}}{d} v_{y}, \quad N_{x}=\beta n_{x}, \quad N_{z}=n_{z}
\end{aligned}
$$

with 


$$
\beta^{2}=\frac{\alpha_{3}}{\alpha_{2}} \frac{K_{22}}{K_{11}} \frac{1}{b}, \quad b=\frac{\eta_{1}}{\eta_{3}},
$$

Eqs.(4) can be rewritten in the form

$$
\begin{array}{ll}
V_{y, z z}-(1-b)\left(\tilde{S} N_{x}\right)_{, z} & =0, \\
\tilde{S} N_{z}+N_{x, z z} & =0, \\
b \tilde{S} N_{x}+N_{z, z z}+V_{y, z} & =0 .
\end{array}
$$

For the shear rate $\tilde{S}$ one has

$$
\begin{array}{ll}
\tilde{S}=a^{2}, a^{2}=\frac{V^{0} \tau_{d}}{d} \beta & \text { - for Couette flow }, \\
\tilde{S}=-a^{2} z, a^{2}=-\frac{\Delta P}{\Delta x} \frac{\tau_{d} d}{\eta_{3}} \beta & \text { - for Poiseuille flow } .
\end{array}
$$

The boundary conditions (5), (7) reduce to

$$
\pm N_{x, z}+w_{a} N_{x}=0, \quad \pm N_{z, z}+w_{p} N_{z}=0, \quad V_{y}=0, \text { at } z= \pm 1 / 2
$$

where

$$
w_{a}=W_{a} d / K_{22}, \quad w_{p}=W_{p} d / K_{11}
$$

In the limit of strong anchoring $\left(w_{a}, w_{p} \rightarrow \infty\right)$ one has $N_{x}=N_{z}=0$ at $z= \pm 1 / 2$ whereas for torque-free boundary conditions $\left(w_{a}, w_{p} \rightarrow 0\right) N_{x, z}=N_{z, z}=0$ at the boundaries. From (14) one can see that by changing the thickness $d$, the dimensionless anchoring strengths $w_{a}$ and $w_{p}$ can be varied, the ratio $w_{a} / w_{p}$ remaining fixed.

Solving the system of linear ordinary differential equations (10) with boundary conditions (13) one can obtain the critical value of the shear rate $a_{c}^{2}$, at which the initial state (1) loses stability, and determine the influence of anchoring strengths $w_{a}$ and $w_{p}$ on $a_{c}^{2}$.

\section{COUETTE FLOW}

In this case the shear rate $\tilde{S}$ is independent of $z$ (11), so that the system (10) can be solved using standard theory of ordinary differential equations with constant coefficients. Further, the $\{z \rightarrow-z\}$ symmetry of Eqs.(10) allows for two possible types of solutions: 


$$
\begin{aligned}
& \text { I: }\left\{V_{y} \text { - even; } N_{x}, N_{z} \text { - odd }\right\} \text { - "odd" solution } \\
& \text { II: }\left\{V_{y} \text { - odd; } N_{x}, N_{z} \text { - even }\right\} \text { - "even" solution . }
\end{aligned}
$$

We will always classify the solutions by the $z$-symmetry of the $x$-component of the director perturbation $N_{x}$. For the odd solution one obtains

$$
\begin{aligned}
& V_{y}=C_{1}(1-b) a \cosh (a z)-C_{2}(1-b) a \cos (a z)+C_{3}, \\
& N_{x}=C_{1} \sinh (a z)+C_{2} \sin (a z), \\
& N_{z}=-C_{1} \sinh (a z)+C_{2} \sin (a z) .
\end{aligned}
$$

Taking into account the boundary conditions (13) the solvability condition for the $C_{i}$ ("boundary determinant" equated to zero) gives the expression for the critical shear rate

$$
\begin{aligned}
& 2 w_{a} w_{p} \sinh \left(a_{c} / 2\right) \sin \left(a_{c} / 2\right)+ \\
& a_{c}\left(w_{a}+w_{p}\right)\left[\cosh \left(a_{c} / 2\right) \sin \left(a_{c} / 2\right)+\cos \left(a_{c} / 2\right) \sinh \left(a_{c} / 2\right)\right]+ \\
& 2 a_{c}^{2} \cosh \left(a_{c} / 2\right) \cos \left(a_{c} / 2\right)=0 .
\end{aligned}
$$

For the even solution one obtains

$$
\begin{aligned}
& V_{y}=C_{1}(1-b) a \sinh (a z)+C_{2}(1-b) a \sin (a z)-C_{3} a^{2} b z \\
& N_{x}=C_{1} \cosh (a z)+C_{2} \cos (a z)+C_{3} \\
& N_{z}=-C_{1} \cosh (a z)+C_{2} \cos (a z) .
\end{aligned}
$$

The boundary determinant condition now gives

$$
\begin{aligned}
& a_{c}^{3} b \sinh \left(a_{c} / 2\right) \sin \left(a_{c} / 2\right)+ \\
& \frac{1}{2} a_{c}^{2} b\left(w_{a}+w_{p}\right)\left[\cosh \left(a_{c} / 2\right) \sin \left(a_{c} / 2\right)-\cos \left(a_{c} / 2\right) \sinh \left(a_{c} / 2\right)\right]- \\
& \left\{a_{c} b \cosh \left(a_{c} / 2\right) \cos \left(a_{c} / 2\right)+\right. \\
& \left.(1-b)\left[\sinh \left(a_{c} / 2\right) \cos \left(a_{c} / 2\right)+\sin \left(a_{c} / 2\right) \cosh \left(a_{c} / 2\right)\right]\right\} w_{a} w_{p}=0 .
\end{aligned}
$$

Note, that the expressions (16) and (18) are both symmetric under exchange of $w_{a}$ and $w_{p}$. This results from the fact that $N_{x}$ and $N_{z}$ have the same symmetry. We found that for MBBA material parameters at $25^{\circ}$ [17] $(b=0.58)$ the critical shear rate for the odd 
mode is systematically higher than for the even mode, so there is no transition between them under variation of the surface anchoring. For strong anchoring $\left(w_{a}=w_{p}=\infty\right)$ the expression for the critical shear rate of the even solution (18) recovers the result obtained by Leslie [7]. Weak surface anchoring reduces the critical shear rate compared to the case of strong boundary conditions. In the limit of one of the surface anchoring strengths going to zero $\left(w_{a} \rightarrow 0\right.$ or $\left.w_{p} \rightarrow 0\right)$ one has $a_{c}^{2} \rightarrow 0$. In figure 1 the critical shear rate $a_{c}^{2}$ for the even solution calculated from Eq.(18) using the material parameters of MBBA $(b=0.58)$ is shown as a function of $1 / w_{a}, 1 / w_{p}$.

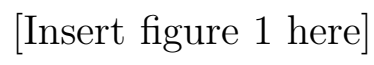

In order to obtain an easy-to-use expression for the critical shear rate of the relevant even mode as a function of the surface anchoring strengths one can use the single-mode approximation in the spirit of a Galerkin expansion [18]. We choose

$$
\begin{aligned}
& V_{y}=C_{1} \sin (2 \pi z), \\
& N_{x}=C_{2}\left[w_{a} \cos (\pi z)+\pi\right], \\
& N_{z}=C_{3}\left[w_{p} \cos (\pi z)+\pi\right],
\end{aligned}
$$

which satisfy boundary conditions (13). Substituting this ansatz into (10) where $\tilde{S}=a^{2}$ and solving the algebraic system for $C_{i},\{i=1,2,3\}$ obtained after projecting the first of Eqs.(10) onto the first mode of Eqs.(19), the second equation onto the second mode etc., one gets

$$
\begin{aligned}
& a_{c}^{2}=3 \pi^{3} \sqrt{w_{a} w_{p}\left(4+w_{a}\right)\left(4+w_{p}\right) /\left(p_{1} p_{2}\right)}, \\
& p_{1}=w_{a} w_{p}+4\left(w_{a}+w_{p}\right)+2 \pi^{2}, \\
& p_{2}=16 w_{a} w_{p}(1-b)+9 \pi^{2} b p_{1} .
\end{aligned}
$$

The single-mode approximation (20) gives almost the same values for $a_{c}^{2}$ as (18) (relative error is smaller than 1\%), so that they could not be differentiated in figure 1. 


\section{POISEUILLE FLOW}

In the case of Poiseuille flow the shear rate $\widetilde{S}$ is given by Eq.(12) and the symmetry properties of system (10) give us the following two types of solutions:

$$
\begin{aligned}
& \text { I: }\left\{V_{y}, N_{x} \text { - odd } ; N_{z} \text { - even }\right\} \text { - "odd" solution , } \\
& \text { II: }\left\{V_{y}, N_{x} \text { - even; } N_{z} \text { - odd }\right\} \text { - "even" solution . }
\end{aligned}
$$

The odd solution corresponds to the splay-mode and the even solution to the twist-mode in the notation of Dubois-Violette and Manneville [9]. Integration of the first equation in system (10) gives

$$
V_{y, z}=K-(1-b) a^{2} z N_{x}
$$

with $K=0$ for the even solution. For the odd solution the integration constant $K$ must be nonzero (except for $b=1$, see below). After eliminating $V_{y, z}$ from the third equation in (10) one arrives at

$$
\begin{aligned}
& a^{2} z N_{z}-N_{x, z z}=0, \\
& a^{2} z N_{x}-N_{z, z z}=K .
\end{aligned}
$$

Following [9] we perform the transformation $Z=a^{2 / 3} z$ and introduce new variables $\mathcal{U}=$ $N_{x}+N_{z}, \mathcal{V}=N_{x}-N_{z}$, leading to

$$
\begin{aligned}
& \mathcal{U}_{, Z Z}-Z \mathcal{U}=-K a^{-4 / 3} \\
& \mathcal{V}_{, Z Z}+Z \mathcal{V}=K a^{-4 / 3}
\end{aligned}
$$

The general solution of (23) can be expressed in terms of Airy functions $A i(Z), B i(Z)$, $G i(Z) \llbracket 19$.

Let us first consider the case of the even solution $(K=0)$. Then one has

$$
\begin{aligned}
& N_{x}=C_{1}[A i(Z)+A i(-Z)]+C_{2}[B i(Z)+B i(-Z)], \\
& N_{z}=C_{1}[A i(Z)-A i(-Z)]+C_{2}[B i(Z)-B i(-Z)],
\end{aligned}
$$


where $C_{1}, C_{2}$ are constants to be determined from the boundary conditions (13). Substitution of (24) into (13) gives the criterion for the threshold shear rate

$$
\frac{w_{a} A i^{+}+a_{c}^{2 / 3} A i_{, Z}^{+}}{w_{a} B i^{+}+a_{c}^{2 / 3} B i_{, Z}^{+}}=\frac{w_{p} A i^{-}+a_{c}^{2 / 3} A i_{, Z}^{-}}{w_{p} B i^{-}+a_{c}^{2 / 3} B i_{, Z}^{-}} .
$$

Here

$$
A i^{ \pm}=A i\left(\frac{1}{2} a_{c}^{2 / 3}\right) \pm A i\left(-\frac{1}{2} a_{c}^{2 / 3}\right), \quad B i^{ \pm}=B i\left(\frac{1}{2} a_{c}^{2 / 3}\right) \pm B i\left(-\frac{1}{2} a_{c}^{2 / 3}\right)
$$

and similar for $A i_{, Z}^{+}$and $B i_{, Z}^{+}$. The limit of strong anchoring $\left(w_{a} \rightarrow \infty, w_{p} \rightarrow \infty\right)$ gives the result of Dubois-Violette and Manneville 99

$$
\frac{A i\left(\frac{1}{2} a_{c}^{2 / 3}\right)}{B i\left(\frac{1}{2} a_{c}^{2 / 3}\right)}=\frac{A i\left(-\frac{1}{2} a_{c}^{2 / 3}\right)}{B i\left(-\frac{1}{2} a_{c}^{2 / 3}\right)},
$$

leading to $a_{c}^{2}=102.59$. The fact that $N_{x}$ and $N_{z}$ have different $z$-symmetry leads in Eq.(25) to an asymmetry in the dependence of the critical shear rate on $w_{a}, w_{p}$, in contrast to the case of Couette flow. The critical shear rate [Eq.(25)] retains a finite value when the polar anchoring strength $w_{p}$ (which mainly controls $N_{z}$ perturbations) vanishes, whereas $a_{c}^{2} \rightarrow 0$ if the azimuthal anchoring strength $w_{a} \rightarrow 0$.

For the odd solution with nonzero $K$ the solution of (22) has the following form

$$
\begin{aligned}
& N_{x}=k K\left\{C_{1} A i^{-}(Z)+C_{2} B i^{-}(Z)+G i^{-}(Z)\right\}, \\
& N_{z}=k K\left\{C_{1} A i^{+}(Z)+C_{2} B i^{+}(Z)+G i^{+}(Z)\right\},
\end{aligned}
$$

where

$$
k=\pi a^{-4 / 3} / 2, \quad A i^{ \pm}(Z)=A i(Z) \pm A i(-Z) \text { etc. }, \text { for } B i^{ \pm}(Z) \text { and } G i^{ \pm}(Z)
$$

The coefficients $C_{i}$ in (27) are determined from the boundary conditions (13). Integrating Eq. (21) and taking into account the boundary conditions $V_{y}(z= \pm 1 / 2)=0$ one obtains the expression for the critical shear rate of the odd mode

$$
K-(1-b) a_{c}^{2} \int_{-1 / 2}^{1 / 2} z N_{x}\left(z ; a_{c}^{2}, w_{a}, w_{p}\right) d z=0
$$


Since $N_{x}$ is proportional to $K$, this undetermined integration constant falls out from (29). From thermodynamical conditions [4, 20] the parameter $b$ has to be positive, but is otherwise not restricted. The point $b=1$ requires special consideration. In this case, from Eq.(21) and boundary conditions for $V_{y}$, it follows that $K=0$ and $V_{y}=0$, so that one should solve Eqs.(22) keeping the symmetry of $N_{x}$ and $N_{z}$ corresponding to the odd solution. In other words, if $b=1$ then one has the homogeneous instability of odd type with zero velocity perturbation, like in the case of Fréedericksz transition. Moreover, in the case $b=1$ Eqs.(22) became invariant with respect to change $\left\{N_{x} \Leftrightarrow N_{z}\right\}$, so that the critical shear rates for even and odd solutions are the same up to transposition $\left\{w_{a} \Leftrightarrow w_{p}\right\}$.

The instability of the odd mode is mainly controlled by the polar anchoring strength $w_{p}$. In the limit of zero azimuthal anchoring strength $w_{a} \rightarrow 0$ one has a finite critical shear rate, whereas $a_{c}^{2} \rightarrow 0$ for the polar anchoring strength $w_{p} \rightarrow 0$.

The critical shear rate $a_{c}^{2}$ for the even solution [Eq.(25)] and odd solution [Eq.(29)] have been calculated for the material parameters of MBBA for various values of $w_{a}, w_{p}$ (figure 2). Depending on the azimuthal and polar surface anchoring strengths one can have different $z$-symmetry of the first unstable mode. The critical line in $\left(w_{a}, w_{p}\right)$ space corresponds to the crossover between the two types of unstable solutions.

[Insert figure 目 here]

Since the expressions (25) and (29) for the critical shear rates of the two possible unstable modes are quite complicated we use a single-mode Galerkin approximation in order to obtain easy-to-use formulas.

For the even mode we can use Eqs.(22) with $K=0$. Assuming

$$
\begin{aligned}
& N_{x}=C_{1}\left[w_{a} \cos (\pi z)+\pi\right], \\
& N_{z}=C_{2}\left[w_{p} \sin (2 \pi z)+2 \pi \sin (\pi z)\right],
\end{aligned}
$$

after substituting (30) into (22) and projection one obtains

$$
\begin{aligned}
& a_{c}^{2}=6 \sqrt{3} \pi^{4} \sqrt{w_{a}\left(4+w_{a}\right)\left(3 \pi^{2}+20 w_{p}+3 w_{p}^{2}\right) / p_{1}^{2}}, \\
& p_{1}=16 w_{a} w_{p}+9 \pi^{2}\left(w_{a}+w_{p}\right)+72 \pi^{2} .
\end{aligned}
$$


For the odd mode we choose

$$
\begin{aligned}
& N_{x}=C_{1}\left[w_{a} \sin (2 \pi z)+2 \pi \sin (\pi z)\right] \\
& N_{z}=C_{2}\left[w_{p} \cos (\pi z)+\pi\right]
\end{aligned}
$$

Substituting this ansatz into (22) one finds the coefficients $C_{1}$ and $C_{2}$. Then from (29) one obtains

$$
\begin{aligned}
& a_{c}^{2}=6 \sqrt{3} \pi^{4} \sqrt{w_{p}\left(4+w_{p}\right)\left(3 \pi^{2}+20 w_{a}+3 w_{a}^{2}\right) /\left(p_{1} p_{2}\right)} \\
& p_{2}=b p_{1}+(1-b)\left(9 \pi^{2}-144-2 w_{a}\right) w_{p}
\end{aligned}
$$

The approximate expressions (31) and (33) give systematically higher values for the critical shear rate compared to (25) and (29), about $10 \%$ for both modes. Equating (31) and (33) one can obtain an approximate expression for the critical line in the $\left(w_{a}, w_{p}\right)$ plane corresponding to the crossover between the critical even and odd modes (see figure 2). We now write $\beta_{a}=1 / w_{a}, \beta_{p}=1 / w_{p}$. For strong azimuthal anchoring $\left(\beta_{a}=0\right)$ the transition from even to odd mode takes place at some critical value of polar anchoring strength: $\beta_{p}=\beta_{p 0}$, where $\beta_{p 0}$ is the solution of the algebraic equation

$$
\begin{aligned}
& 9 \pi^{4} b \beta_{p 0}^{3}+2 \pi^{2}(39 b-19) \beta_{p 0}^{2}+ \\
& {\left[-9 \pi^{2}(1-b)+120 b-\frac{232}{3}\right] \beta_{p 0}-18(1-b)=0 .}
\end{aligned}
$$

For MBBA material parameters one finds $\beta_{p 0}=0.307$. Assuming the deviation $\left(\hat{\beta}_{a}, \hat{\beta}_{p}\right)$ from $\left(0, \beta_{p 0}\right)$ to be small one has up to first order in $\hat{\beta}_{a}, \hat{\beta}_{p}$ for the critical line

$$
c_{1}\left(\beta_{p 0}+\hat{\beta}_{p}\right)+c_{2} \hat{\beta}_{a}=0
$$

where

$$
\begin{aligned}
c_{1} & =27 b \pi^{4} \beta_{p 0}^{2}+4 \pi^{2}(39 b-19) \beta_{p 0}-9 \pi^{2}(1-b)+120 b-\frac{232}{3} \\
c_{2} & =108 b \pi^{4} \beta_{p 0}^{3}+\pi^{2}\left(9 b \pi^{2}+936 b-680\right) \beta_{p 0}^{2}-36\left(3 \pi^{2}+40\right)(1-b) \beta_{p 0}+ \\
& +216 b-\frac{776}{3} .
\end{aligned}
$$

The results for the critical line for the crossover between even and odd critical modes obtained from the numerical solutions of Eqs.(25), (29) and approximation (35) are shown in figure 3 . 
[Insert figure 3 here]

Note, that $\beta_{a}=1 / w_{a}, \beta_{p}=1 / w_{p}$ are inversely proportional to the thickness $d$ of the NLC layer. Therefore, varying the cell thickness, one can cross the critical line separating the two regimes. For that purpose it is necessary to have the ratio $\beta_{p} / \beta_{a} \equiv K_{11} W_{a} /\left(K_{22} W_{p}\right)$ larger than the slope of the critical line $\left(\sim-c_{2} / c_{1}\right)$. Using the material parameters of MBBA one obtains $W_{a} / W_{p}>-c_{2} K_{22} /\left(c_{1} K_{11}\right)=2.28\left(W_{a} / W_{p}>1.78\right.$ from numerical results). For small values of $w_{a}$ and $w_{p}$, the crossover is given by $w_{a}=w_{p} / b$. As was said above, if $b=1$, Eqs. (22) are invariant with respect to $\left\{N_{x} \Leftrightarrow N_{z}\right\}$. This means that the crossover line for $b=1$ is exactly defined by $w_{a}=w_{p}$. The same result follows from approximation formulas (31) and (33).

\section{CONCLUSIONS AND DISCUSSION}

It was found that changes of the anchoring strengths can cause a crossover between two types of homogeneous instabilities induced by steady Poiseuille flow, in contrast to the case of steady Couette flow, where the first unstable mode is always the even one. Semi-analytical expressions for the critical shear rates for both Poiseuille and Couette flow are presented, together with simple approximate formulas of good accuracy.

The effect of the reduction of the critical shear rate for homogeneous instabilities under weak surface anchoring can in principle be used for the determination of the polar $\left(W_{p}\right)$ and azimuthal $\left(W_{a}\right)$ anchoring strengths. For that purpose one may measure the critical shear

rates for two cells with different thicknesses and use the full expressions (or approximate formulas) to calculate both $W_{p}$ and $W_{a}$. Alternatively, one can use an additional electric field applied across the NLC cell. Depending on the sign of the dielectric anisotropy of the NLC the electric field will stabilise or destabilise $n_{z}$ fluctuations and affect the critical shear rate differently for different values of the polar and azimuthal anchoring strengths. This study is in progress. The advantage of using measurements of the critical shear rate for the determination of polar and azimuthal anchoring strengths compared to the other methods 
(Fréedericksz transition, orientational transition with hybrid orientation, small-angle light scattering) is that here one does not need to modify (or rebuild) the experimental setup in order to obtain $W_{p}$ and $W_{a}$ at the same time.

It would be particularly interesting to investigate experimentally the orientational behaviour of NLC under steady Poiseuille flow for a cell with $W_{p}, W_{a}$ close to the crossover line separating critical modes of different symmetry.

\section{ACKNOWLEDGEMENTS}

Financial support of Deutscher Akademischer Austauschdienst (DAAD), DFG (Grant Kr690/14-1) and INTAS (Grant 96-498) are gratefully acknowledged. O.T. and A.K. wish to thank the University of Bayreuth for its hospitality. 


\section{REFERENCES}

[1] Chigrinov, V.G., 1999, Liquid Crystals Devices: Physics and Applications, (NewYork: Artech House)

[2] Khazimullin, M.V., Börzsönyi, T., Krekhov, A.P., and Lebedev, Yu.A., 1999, Mol. Cryst. Liq. Cryst., 329, 247-254

[3] Nasibullayev, I.Sh., Krekhov, A.P., and Khazimullin, M.V., Mol. Cryst. Liq. Cryst., in press

[4] Leslie, F.M., 1979, Adv. Liq. Cryst., 4, 1-81

[5] Dubois-Violette, E., and Manneville, P., 1996, Flow instabilities in nematics. In Pattern Formation in Liquid Crystals, edited by A. Buka and L. Kramer (Berlin: SpringerVerlag), pp. 91-164

[6] Krekhov, A.P., Börzsönyi, T., Tóth, P., Buka, Á., and Kramer, L., 2000, Physics Reports, 337, 171-192

[7] Leslie, F.M., 1976, J. Phys. D: Appl. Phys., 9, 925-937

[8] Manneville, P., and Dubois-Violette, E., 1976, J. de Phys. (France), 37, 285-296

[9] Manneville P., and Dubois-Violette, E., 1976, J. de Phys. (France), 37, 1115-1124

[10] Manneville, P., 1979, J. de Phys. (France), 40, 713-724

[11] Pieranski, P., and Guyon, E., 1974, Phys. Rev. A, 9, 404-417

[12] Pieranski, P., and Guyon, E., 1973, Sol. St. Comm., 13, 435-437

Pieranski, P., and Guyon, E., 1976, Comm. Phys, 1, 45-49

[13] Dubois-Violette, E., Gyuon, E., Janossy, I., Pieranski, P., and Manneville, P., 1977, J. de Mec., 16, 734-767

[14] Janossy, I., Pieranski, P., and Guyon, E., 1976, J. de Phys. (France), 37, 1105-1113

[15] Guyon, E., and Pieranski, P., 1975, J. de Phys. (France), 36, C1-203-C1-208

[16] Pikin, S.A., 1974, Sov. Phys. JETP, 38, 1246-1250

Pieranski, P., Gyuon, E., and Pikin, S.A., 1976, J. de Phys. Colloq. Paris, 37, C1-3-C1-6 
Cladis, P.E., and Torza, S., 1975, Phys. Rev. Lett., 35, 1283-1286

[17] Kneppe, H., Schneider, F., and Sharma, N.K., 1982, J. Chem. Phys., 77, 3203-3208 de Jeu, W. H., Claassen, W. A. P., and Spruijt, A. M. J., 1976, Mol. Cryst. Liq. Cryst., 37, 269-280

[18] Gottlieb, D., and Orszag, S.A., 1993, Numerical analysis of spectral methods: theory and applications, (Montpelier: Capital City Press)

Pesch, W., and Kramer, L., 1996, General mathematical description of pattern-forming instabilities, In Pattern Formation in Liquid Crystals, edited by A. Buka and L. Kramer (Berlin: Springer-Verlag), pp. 69-90

[19] see, e.g., Abramowitz, M., and Stegun, I.A., 1964, Handbook of Mathematical Functions, (New York: Dover), Chap. 10

[20] Pleiner, H., and Brand, H. R., 1996, Hydrodynamics and electrohydrodynamics of liquid crystals, In Pattern Formation in Liquid Crystals, edited by A. Buka and L. Kramer (Berlin: Springer-Verlag), pp. 15-68 


\section{FIGURES}

FIG. 1. Critical shear rate of Couette flow vs. anchoring strengths. $b=0.58$ (MBBA).

FIG. 2. Critical shear rate of Poiseuille flow vs. anchoring strengths. $b=0.58$ (MBBA).

FIG. 3. Critical line separating odd and even regimes in Poiseuille flow: numerical solution (solid) and approximation (35) (dotted). $b=0.58$ (MBBA). 


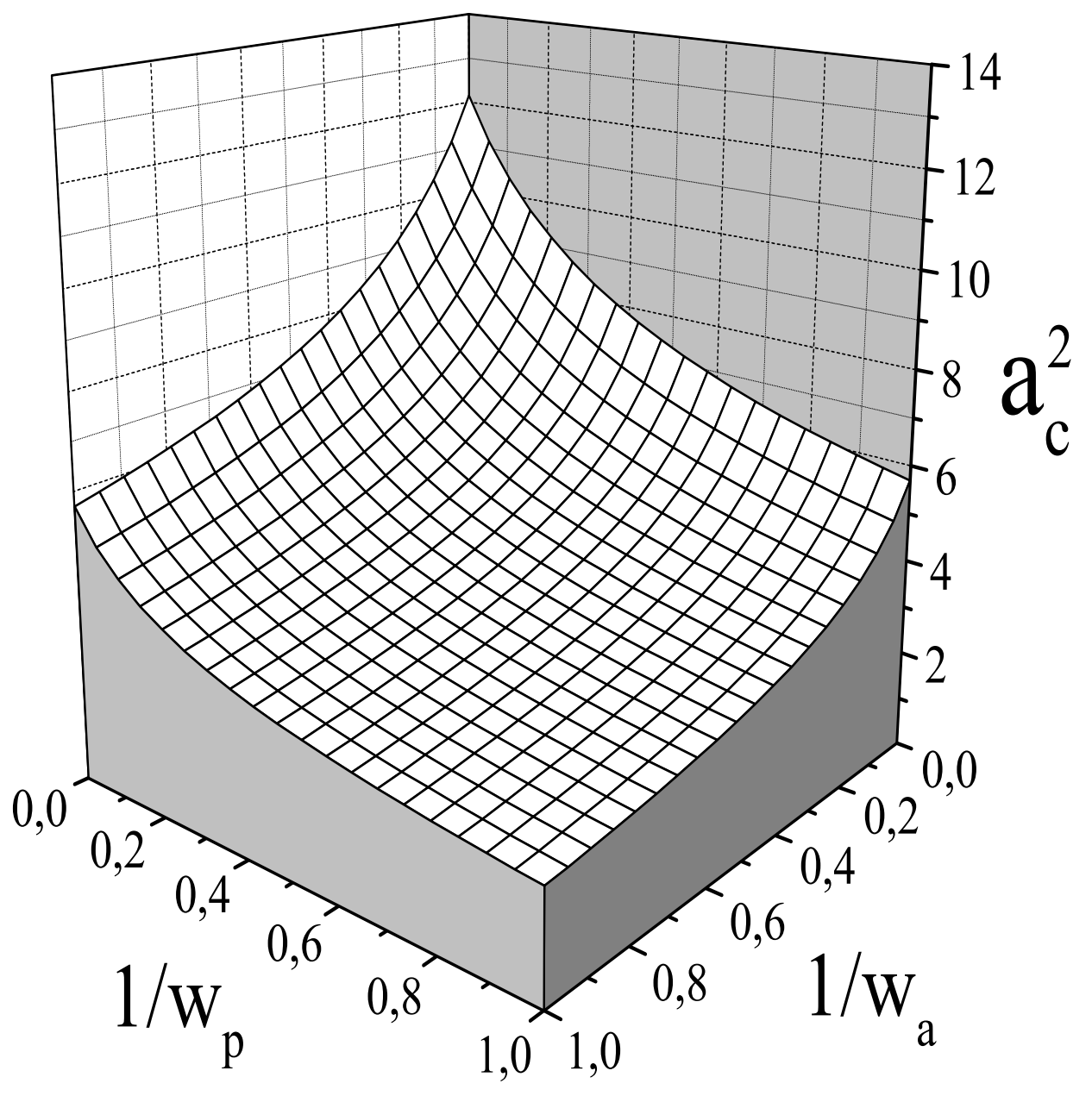




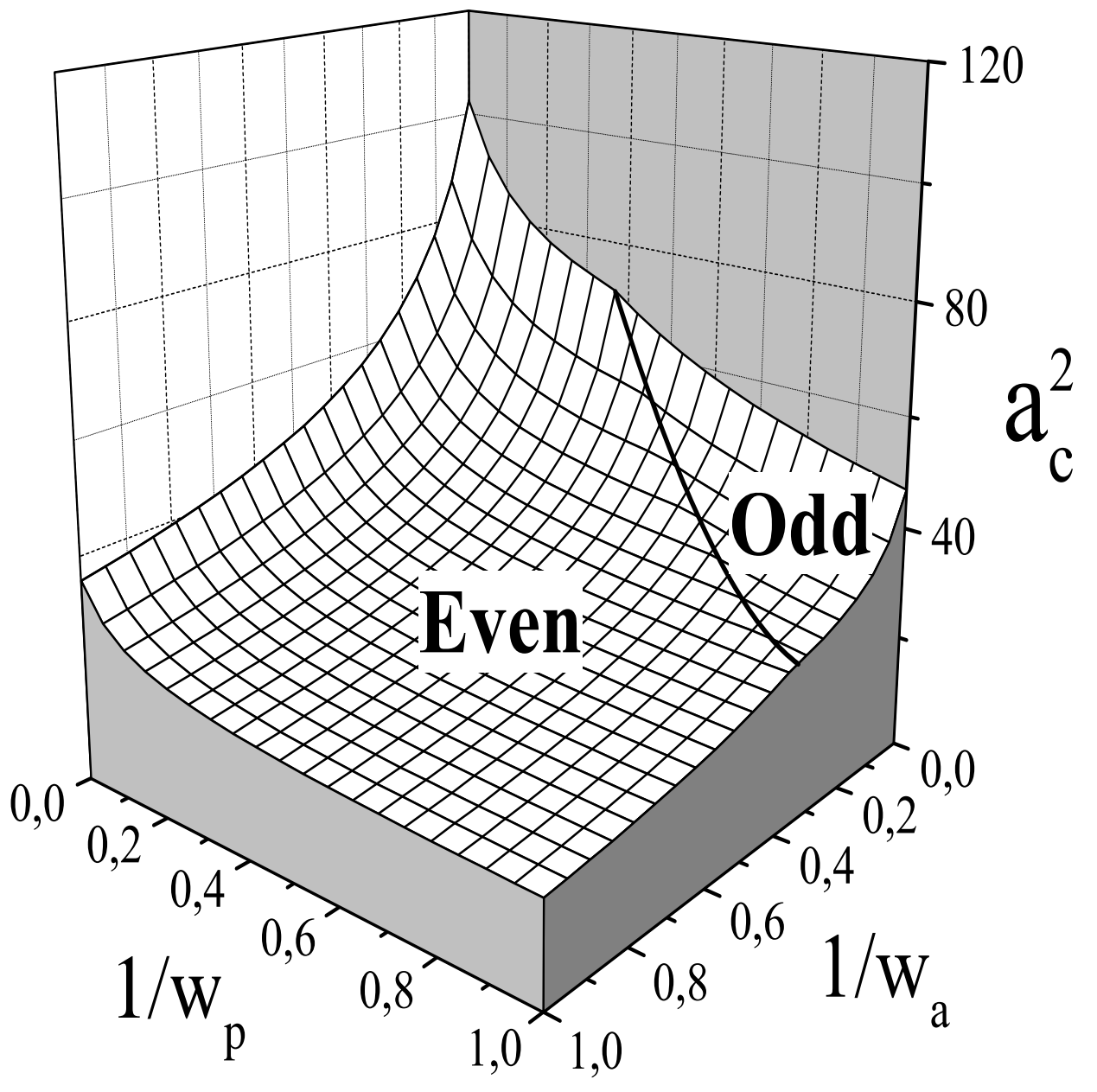




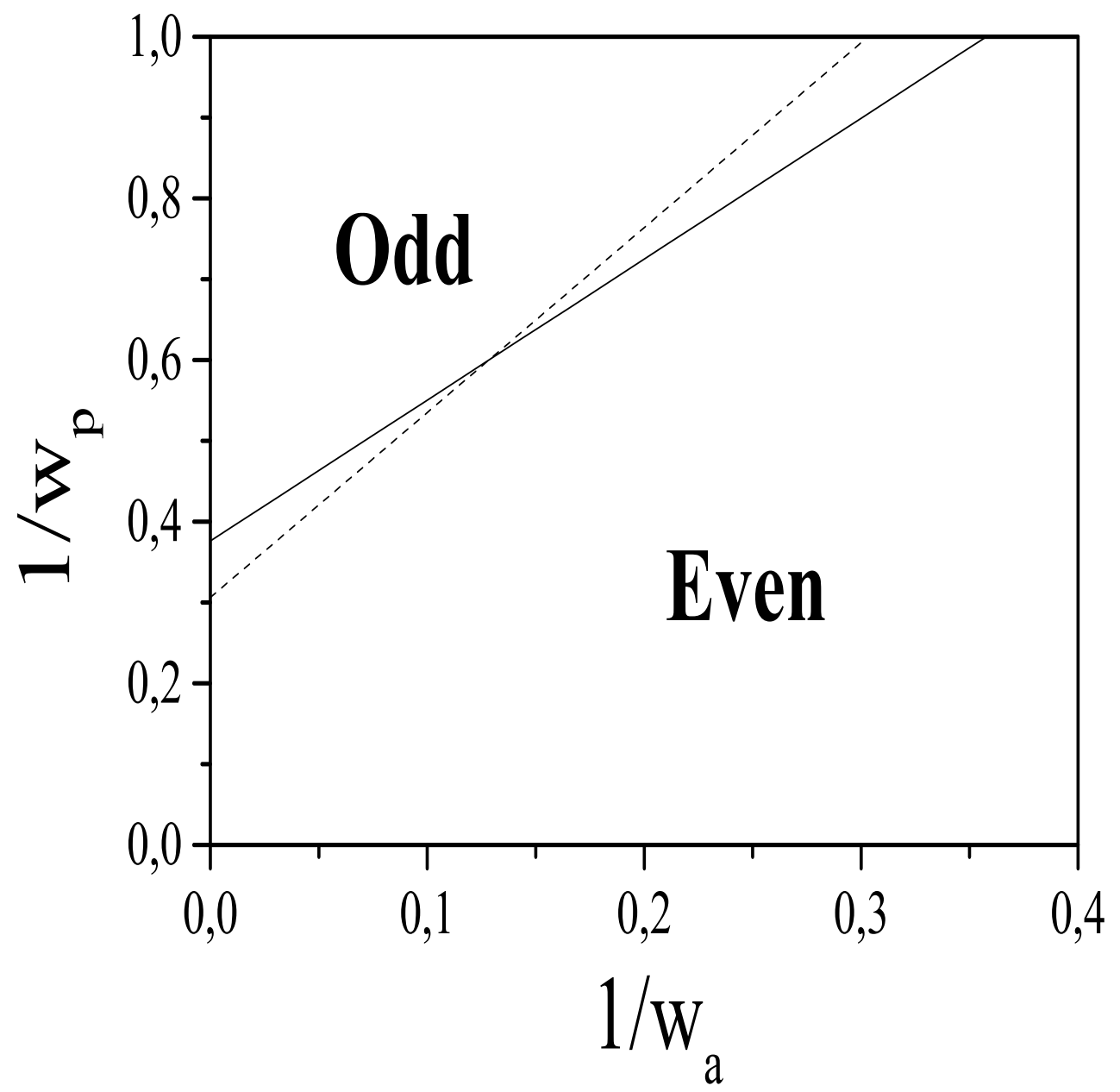

